\title{
Current Concepts of Bone Regeneration in Implant Dentistry
}

\author{
Reena Rodriguez*, Nico Hartmann and Dieter Weingart \\ Department of Oral and Maxillofacial Surgery, Katharinen Hospital, Klinikum Stuttgart, Germany
}

\begin{abstract}
The use of dental implants for the rehabilitation of missing teeth has broadened the treatment options for patients and clinicians equally. As a result of advances in research in implant design, materials and techniques the use of dental implants has increased dramatically in the past two decades and is expected to expand further in the future. However the clinical complexity of the patient who present with limited bone volume often requires additional biomaterials and surgical procedures to ensure successful implant treatment. This review outlines the various biomaterials used in augmenting bone deficiencies encountered and the different surgical techniques that are used in order to achieve a predictable long term success of dental implants.
\end{abstract}

Keywords: Dental implants; Bone regeneration; Current concepts

\section{Introduction}

Success of dental implants depends largely on the quality and quantity of available bone in the recipient site [1]. This however may be compromised or unavailable due to tumor, trauma or periodontal disease etc., which in turn necessitates the need for additional bone augmentation. Successful regeneration requires the essential components of cells, scaffold and signaling molecules. This is effectively achieved with the use of autologous bone and is thus considered the gold standard of bone regeneration against which all other types of bone regenerative materials are compared. However autologous bone grafting is associated with morbidity of the recipient site. With the tremendous advances in the field of regenerative biomaterials, a wide range of biomaterials have been tested as an alternative to autologous bone. This review outlines the present scenario in bone regenerative therapy in implant dentistry, the various biomaterials, surgical techniques employed to circumvent different clinical situations and the future directions in the field of bone regeneration.

\section{Autogenous bone grafts}

One of the most difficult challenges in implant dentistry is the treatment of large bone defects or deficiencies. Autologous bone offers a wide pool of growth factors that can induce mesenchymal stem cells to differentiate into osteogenic progenitor cells [2]. They also help in the osteoconduction by acting as a scaffold on which further apposition may occur [3]. Furthermore, there is no risk of immune rejection and the long term studies indicate excellent long term results. The preferred site for intraoral bone harvesting is the retromolar, angle of the mandible and chin. Extra orally the iliac crest and tibia are employed for bone harvesting. The quantity of autologous bone however may often be limited to avoid extensive morbidity of the donor site. Several techniques have been developed in order to circumvent this problem [4-6]. They maybe by the use of a modified surgical technique, volume expansion of the autologous graft or by the addition of a non allogenic graft material.The ultimate decision for the choice of the graft material is dictated by the complexity of the deficiency and the approval of the treatment option by the patient.

\section{Allograft}

An allograft is a tissue graft between individuals of the same specimen but of non-identical genetic composition. The source is usually cadaver bone, which is subjected to a treatment sequence which renders it neutral to immune reactions and helps to avoid cross contamination of host diseases. Human bone material in the form of freeze dried bone or demineralised freeze dried bone (DFDB) has been used in periodontology and implant dentistry. The disadvantages are an increased risk of immunogenicity, a rather quicker resorption rate compared to autogenous bone and a risk of disease transmission [6].

\section{Xenografts}

Xenografts are tissue grafts between two different species. Deproteinized bovine bone matrix (DBBM) is a well-documented bone substitute for intraoral bone grafting [7]. Other materials in use are of coral source and porcine sources.

\section{Alloplast}

Graft material which is synthetically derived and do not originate from humans or animals. Calcium phosphate materials have attracted particular interest due to the similiarity in the mineral composition of natural bone. Materials such as hydroxyapatite and alpha tricalcium phosphate, beta tricalcium phosphate have widely been used as fillers on their own or combined with autogenous bone [8]. They provide an osteoconductive scaffold for the osteogenic cells but are not by themselves osteoinductive. The use of alloplastic grafting materials on their own is not routinely recommended. However, their use with bone promoting agents has been widely studies in the recent years with predictable outcome.

\section{Growth factors}

Various growth factors have widely been tested in animal models. Of these, Bone Morphogenic Proteins (BMPs) requires special mention as they induce osteogenic precursor cells into osteogenic cells and have shown tremendous bone growth in many animal and

*Corresponding author: Reena Rodriguez, Department of Oral and Maxillofacia Surgery, Katharinen Hospital, Klinikum Stuttgart, Kriegsbergstrasse 60, D 70174 Stuttgart, Germany, Tel: 0711-2783301; Fax: 0711-2783309; E-mail: drreenajoseph@gmail.com

Received December 03, 2014; Accepted February 17, 2015; Published February 22, 2015

Citation: Rodriguez R, Hartmann N, Weingart D .Current Concepts of Bone Regeneration in Implant Dentistry. Journal of Surgery [Jurnalul de chirurgie] 2015 10(4): 263-265 DOI:10.7438/1584-9341-10-4-4

Copyright: @ 2015 Rodriguez R, et al. This is an open-access article distributed under the terms of the Creative Commons Attribution License, which permits unrestricted use, distribution, and reproduction in any medium, provided the original author and source are credited. 
also human clinical studies. Extensive research is being undertaken to develop injectable formulations for minimally invasive application with novel carriers for prolonged and targeted local delivery [9]. Other growth factors besides BMPs that have been implicated during bone regeneration are also being investigate [10], including platelet-derived growth factor, transforming growth factor- $\beta$, insulin-like growth factor-1, vascular endothelial growth factor and fibroblast growth factor, among others [11].

\section{Guided bone regeneration}

Guided bone regeneration (GBR) also known as "membrane protected bone regeneration" refers to the use of barrier membranes in the treatment of alveolar ridge defects. It helps to separate the hard tissue compartment (bone, bone marrow and bone defect) from the soft tissue compartment thereby enabling bone regeneration by space creation [12]. It also effectively stabilizes the blood coagulum and thereby allows for faster healing to occur. This technique can be used before or at the same time as implant placement. Barrier membranes may be non-resorbable (eg: expanded poly tetrafluoroethelene e-PTFE) or resorbable. Although non-resorbable have shown the most bone volume gain, they are associated with a higher incidence of complications such as membrane exposure due to soft tissue dehiscence [13]. The different techniques for bone augmentation are as below:

\section{Graft and membrane}

Depending on the amount of localized deficiency, implants may be placed at the same stage as augmentation (1 step surgery) or following bone regeneration (2 step surgery).

When the localized deficiency is a dehiscence or fenestration type, particulate autograft or bone substitute covered with a membrane is indicated. Horizontal and vertical ridge augmentation procedures often require the use of autologous bone block graft combined with a membrane or a particulate autograft with a bone substitute and a membrane. A healing period of 3 to 6 months before the second stage surgery is undertaken is recommended as delays greater than 6 months may result in resorption of the graft.

\section{Onlay grafts}

Onlay grafts have been used with great success and predictability in the augmentation of severely resorbed edentulous ridges for the treatment with dental implants [6]. Both intra oral (mental symphysis, body of the mandible and ramus, maxillary tuberosity) and extra oral (iliac crest, calvarium,tibia)donor sites may be used depending on the amount of bone that is required for reconstruction. Modelling of the bone graft and fixation of the grafts is done by miniscrews or miniplates and the use of particulated autograft and other forms of bone substitutes maybe used in combination with a bioresorbable membrane (Figure1). A tension free closure of the overlying flap is also critical for the success of the augmentation.

\section{Maxillary sinus lift using lateral approach}

The loss of posterior maxillary teeth causes resorption of the alveolar process in the oral side, by expansion of the sinus cavity into the alveolar process, or both these methods. This results in a lack of sufficient quality and quantity of bone for implant placement. Bone deficiencies in the posterior maxillary regions are one of the most challenging situations in implant dentistry. This can be circumvented with bone augmentation following a sinus floor elevation which involves elevating the schneiderian membrane from the maxillary sinus floor (Figure 2). This technique is often used as a pre-implant procedure when the residual alveolar ridge has is inadequate to a point where initial implant stability is compromised. Implant placement can be done after 3-6 months. Maxillary sinus grafting may also be combined with nasal inlay grafting if the bone volume in the sub nasal area for placement of implants also needs to be increased.

\section{Maxillary sinus elevation by using the transalveolar approach}

This method is less invasive and involves moving the sinus floor by gently fracturing it with the use of osteotomes [14]. Maxillary sinus elevation using the transalveolar approach can be recommended in sites with adequate alveolar crest width, initial bone height of at least 5 $\mathrm{mm}$ and a relative flat sinus floor anatomy.

\section{Split-Ridge /Ridge -expansion technique}

Split ridge/ridge expansion technique refers to the creation of a linear groove in the middle of the ridge with rotary burs or a piezosurgery device and deepening this groove with an osteotome chisel. The lingual or palatal cortical bone is used as a guide and careful tapping with a mallet will advance the chisel into the cancellous part of the bone $[15,16]$.

This technique is indicated in cases of the atrophy of the edentulous ridge has developed horizontally and the cancellous bone is present between the oral and facial cortical plates and the presence of adequate residual height exists. A split ridge with conventional implant placement or a split ridge with interspersed bone graft material may be used.

\section{Vertical distraction osteogenesis}

It is the creation of new bone and adjacent soft tissue after gradual and controlled displacement of a bone fragment obtained by surgical osteotomy. This technique is used to increase the height of the alveolar ridge. It is indicated in cases with vertical deficient ridges (minimum 3 $\mathrm{mm}$ height) with adequate residual width [6]

\section{Le-fort I Osteotomy with interpositional autologous bone grafts}

This is a technique indicated in cases of extremely severe alveolar ridge resorption and there is an unfavourable intermaxillary relationship in the horizontal and vertical plane [6].

\section{Discussion and Conclusion}

The recent advancements in nanotechnology and biomaterial engineering have led to several advancements in the field of implantology and regenerative medicine. An improved implant design, surface and material modification at a nanoscale have greatly contributed in the success and predictability of dental implant treatment [17]. Various bone regenerative materials in the form of gels, particle and scaffolds have also been designed with the help of
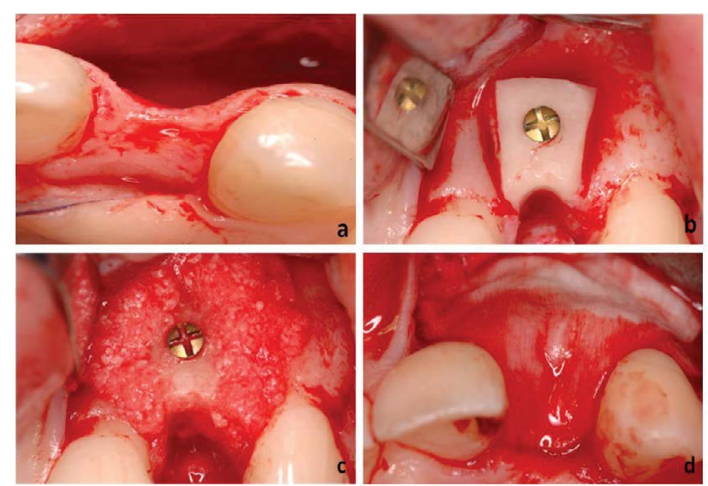

Figure 1:

a.Extensive alveolar ridge atrophy in left lateral incisor region.

b.Bone block augmentation with transplant from angle of the mandible.

c.Bone augmentation using autologous bone particles

d.Guided bone regeneration with Biogide membrane. 

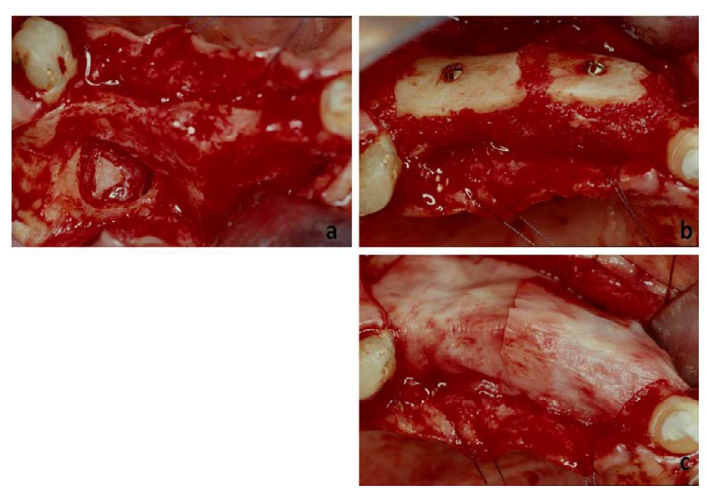

Figure 2:

a. Osteoplastic flap elevation to access the maxillary sinus for an external sinus lift in region 24-26.

b. Horizontal mandibular augmentation with autologous bone block transplant from the angle of the mandible and fixation with titanium screws.

c. Adaptation of Biogide membrane.

nanotechnology and engineering and have opened up a new horizon for bone regeneration [18].

Many studies have shown that nanometer-controlled surfaces can influence early events such as the adsorption of proteins, blood clot formation, and cellular migration and differentiation of mesenchymal stem cells [19-21]. Novel drug delivery systems are being studied for the local delivery of compounds that can enhance osseointegration and bone regeneration [22]. However, several factors such as resorption rate and drug release kinetics are important considerations. An optimum release of the drug is important as the development of tissues is orchestrated by the coordinated interactions of multiple growth factors along spatial and temporal gradients.

In tissue engineering, mesenchymal stem cells (MSCs) are isolated from the patient, expanded and seeded onto a synthetic scaffold and allowed to produce extracellular matrix on the scaffold in controlled culture conditions. This is finally implanted into the bone defect in the patient. The clinical significance of tissue engineering lies in our ability to predictably direct cells to differentiate into the right phenotypes in a spatially and temporally defined pattern.

However, the cost, technical difficulties, failure of the tissue engineered bone to vascularize are still impediments for the routine use of tissue engineered bone in the clinical situation.

The clinical outcome of any regenerative procedure is a combination of the influences from systemic factors, the osteogenic, osteoinductive and osteoconductive properties of the biomaterial, as well as the regenerative potential of the surrounding tissue. The clinician should therefore be mindful to choose the biomaterial based on the 1.diagnosis of the overall health condition of the patient, 2.complexity of the clinical situation at hand and also 3.the long term clinical results of the biomaterial and use the treatment protocol that are simple, involves the least risk with minimal intervention.

\section{Conflict of interests}

Authors have no conflict of interests to disclose.

\section{References}

1. Adell R, Lekholm U, Rockler B, Branemark PI (1981) A 15-year study of osseointegrated implants in the treatment of the edentulous jaw. Int J Oral Surg 6: 387-416.

2. Ferrer MS, Michurina TV, Ferraro F, Mazloom AR, MacArthur BD, et al. (2010) Mesenchymal and haematopoietic stem cells form a unique bone marrow niche. Nature, 466: 829-834.
3. Lichte P, Pape HC, Pufe T, Kobbe P, Fischer H (2011) Scaffolds for bone healing: concepts, materials and evidence. Injury 42: 569-573.

4. Misch CM (1996) Ridge augmentation using mandibular bone graft for the placement of dental implants: Presentation of a technique. Practical Perio and Aest 8: 127-135.

5. Buser D, Dula K, Belser UC, Hirt HP, Berthold H (1995) Localized ridge augmentation using guided bone regeneration. II. Surgical Procedure in the mandible. Int J Periodont Res Dent 15: 11-29.

6. Weingart $D$ ten Bruggenkate CM (2000), Treatment of fully edentulous patients with ITI implants. Clin Oral Implants Res 11: 69-82.

7. Ehrler DM, Vaccaro AR (2000) The use of allograft bone in lumbar spine surgery. Clin Orthop Relat Res 371: 38-45.

8. Esposito M, Grusovin MG, Kwan S, Worthington HV, Coulthard P (2008) Inteventions for replacing missing teeth: bone augmentation techniques for dental implant treatment. Cochrane Database Syst Rev 16: CD003607.

9. Nauth A Giannoudis PV, Einhorn TA, Hankenson KD, Friedlaender GE,et al (2010) Growth factors: beyond bone morphogenetic proteins. J Orthop Trauma 24: 543-546.

10. Simpson AH, Mills L, Noble B (2006) The role of growth factors and related agents in accelerating fracture healing.J Bone Joint Surg Br 88: 701-705.

11. Dimitriou R, Tsiridis E, Giannoudis PV (2005) Current concepts of molecular aspects of bone healing. Injury 36: 1392-1404.

12. Kay S, Wisner-Lynch L, Marxer M, Lynch SE (1997) Guided bone regeneration: Integration of Resorbable Membrane and a bone graft material. Pract Periodontics Aesthet Dent 9: 185-194.

13. Jensen SS, Terheyden H (2009) Bone augmentation procedures in localized defects in the alveolar ridge: clinical results with different bone grafts and bonesubstitute materials. Int J Oral Maxillofac Implants 24: 218-236.

14. Wheeler SL (1997) Sinus augmentation for dental implants: The use of alloplastic materials. J Oral Maxillofac Surg 55: 1287-1293.

15. Lustmann J, Lewinstein I (1995) Interpositional bone grafting technique to widen narrow maxillary ridge. Int J Oral Maxillofac Implants 10: 568-577.

16. Engelke WG, Diederichs CG, Jacobs HG, Deckwer I (1997) Alveolar reconstruction with splitting osteotomy and microfixation of implants. Int J OralMaxillofaclmplants 12: 310-318

17. Albrektsson T, Wennerberg A (2004) Oral implant surfaces: part 1 - review focusing on topographic and chemical properties of different surfaces and in vivo responses to them. Int J Prosthodont 17: 536-543.

18. Albrektsson T, Wennerberg A (2004) Oral implant surfaces: part 2 - review focusing on clinical knowledge of different surfaces Int J Prosthodont 17: 544-564.

19. Anselme K, Bigerelle M (2005) Topography effects of pure titanium substrates on human osteoblast long-term adhesion. Acta Biomater 1: 211-222.

20. Roux C, Fechtenbaum J, Kolta S, Isaia G, Andia JB, et al. (2008) Strontium ranelate reduces the risk of vertebral fracture in young postmenopausal women with severe osteoporosis. Annals of the Rheumatic Diseases 67: 1736-1738.

21. Wennerberg A, Ektessabi A, Albrektsson, T, Johansson C, Andersson, B (1997). A 1-year follow-up of implants of differing surface roughness placed in rabbit bone. Int J Oral Maxillofac Implants 12: 486-494.

22. Zhang L, Webster TJ (2009) Nanotechnology and nanomaterials: promises for improved tissue regeneration. Nano Today 4: 66-80. 